\title{
Nine years old child with nephrotic syndrome: a case of biopsy proven lupus nephritis in tertiary level medical college hospital in Mymensingh, Bangladesh.

\author{
*Hasan $\mathrm{MJ}^{1}$, Ray NC ${ }^{2}$, Quddus ASMR ${ }^{3}$
}

\section{Abstract}

We observed a case of a 9 years old female patient who presented with nephrotic syndrome and impaired renal function diagnosed to have Systemic Lupus Erythematosus (SLE). Lupus nephritis (LN) is a common major organ manifestation of SLE and causes significantly increased morbidity and mortality. Thus, all patients with SLE should be regularly screened for LN. This patient presented with nephrotic syndrome, pleural effusion and pericardial effusion which depicts the multisystem effects of SLE. This patient was treated with Oral Mycophenolate Mofetilin combination with steroid as induction therapy and attained remission after a month of treatment. Systemic lupus erythematosus should be considered in patients with nephrotic syndrome and these patients should have renal biopsy to determine renal involvement.

CBMJ 2018 January: vol. 07 no. 01 P: 40-44

Keywords: Nephrotic syndrome, Lupus nephritis, Systemic lupus erythermatosus,

\section{Introduction}

Lupus nephritis (LN) is a major cause of morbidity and mortality in patients with systemic lupus erythematosus (SLE). The general consensus is that $60 \%$ of lupus patients will develop clinically relevant nephritis at some time in the course of their illness. ${ }^{1}$ Prompt recognition and treatment of renal disease is important; as early response to therapy is correlated with better outcome. ${ }^{2}$ Most SLE patients develop nephritis early in the course of their disease. The vast majority of patients who develop nephritis are younger than 55 years, and children are more likely to develop severe nephritis than are elderly patients. ${ }^{3}$

In a recent retrospective study, male sex, young age (<33 years) and non-European ancestry were found to be determinants of earlier renal disease in patients with SLE. Asian, African Caribbean, and African American ethnicities may present with more severe nephritis than other ethnic groups. ${ }^{4}$

Proteinuria is the characteristic feature of renal disease in lupus. In a comprehensive review of LN, proteinuria was reported in $100 \%$ of patients, with nephrotic syndrome being reported in 45 to $65 \%{ }^{5}$ Occurrence of Microscopic hematuria was found in about $80 \%$ of patients during the disease course, invariably associated with proteinuria. Macroscopic hematuria is rare in LN. Hypertension is not common but is present more frequently in patients with severe nephritis. About one-half of all patients with LN will have a reduced glomerular filtration rate and occasionally patients with acute kidney injury.

The diagnosis of lupusnephritis is usually considered in patient with SLE presenting with features indicating renalinvolvement, proteinuria, haematuria, red blood cell casts and elevated serum creatinine. ${ }^{6}$

This report describes a patient who presented with nephrotic syndrome to Department of Nephrologyat Community Based Medical College Hospital, winnerpar, Mymensingh.

1. Dr. Mahmud Javed Hasan, Associate professor and Head, Department of Nephrology, Community Based Medical College Bangladesh

2. Dr. Nitai Chandra Ray, Assistant Professor, Department of Nephrology, Community Based Medical College Bangladesh

3. Prof. Dr. A.S.M. Ruhul Quddus, Professor (C.C), Department of Pediatrics, Community Based Medical College, Bangladesh

Address of correspondence:

Email:dr.porag@gmail.com

Mobile:01712177065 


\section{Case report}

A 9 years old female was admitted in the Department of Nephrologyat Community Based Medical College Hospital, winnerpar, Mymensingh with history of joint pain and generalized body swelling for 6 months, skin lesions for one month. Joint pain was involving multiple big joints; there was no history of joint swelling or morning stiffness. Body swelling started on the face and was worse in the morning subsiding towards the evening. Swelling also involved abdomen and the lower limbs. There was history of frothy urine. However, no history of haematuria or reduced urine output was given. The patient reported history of dyspnoea especially on lying flat and worse on exertion three weeks after generalized body swelling. One month prior to admission, the patient developed itching generalized hyperpigmented skin lesions which did not affect the face.

On physical examination, she was wasted with light and easily pluck-able sparse hair with patchy alopecia. She had some palmar pallor with pedal oedema and no lymphadenopathy. Respiratory rate was 36 breaths/ minute and trachea was central. She had reduced tactile vocal fremitus bilaterally in the infra-mammary and infrascapular positions and reduced breath sounds consistent with bilateral pleural effusion. Pulse rate was 106 beats/minute, blood pressure was 140/95 $\mathrm{mmHg}$, apex beat with difficult to locate and heart sounds were muffled, these features were consistent with pericardial effusion. Patient had hyper-pigmented lesions on the abdominal skin, ascites and hepatomegaly of $12 \mathrm{~cm}$ span.

Urinalysis revealed proteinuria +3 , twenty four hours urinary protein excretion was $6.8 \mathrm{~g}$, serum albumin was $15 \mathrm{~g} / \mathrm{L}$, and serum cholesterol was $6.24 \mathrm{mmol} / \mathrm{L}$. Serum creatinine was $1.6 \mathrm{mg} / \mathrm{dl}$, serum urea was $115 \mathrm{mg} / \mathrm{dl}$, glomerular filtration rate was estimated to be $34 \mathrm{ml} / \mathrm{min}$. Serum electrolytes revealed hyperkalaemia (5.9 $\mathrm{mmol} / \mathrm{L})$ and hyponatremia (128 $\mathrm{mmol} / \mathrm{L})$. Haemoglobin level was $7.9 \mathrm{~g} / \mathrm{dL}$ with mean corpuscular volume and mean corpuscular haemoglobin concentration of $77.6 \mathrm{fL}$ and
$24 \mathrm{pg}$ respectively. Platelet count was 490 $x$ 103/ $\mu \mathrm{L}$. HIV, hepatitis B and C screening were negative. Anti-nuclear antibody test (ANA) and anti-double stranded DNA (antidsDNA) were both positive. Abdominal ultrasound revealed normal sized kidneys, grade one echogenicity and mild loss of cortical-medullary differentiation. Chest $\mathrm{x}$ ray revealed bilateral pleural effusion, electrocardiogram showed low voltage and echocardiogram revealed pericardial effusion.

Renal biopsy was performed after stabilizing the patient and light microscopy slide with Haematoxylin and Eosin, Periodic acid Schiff and Silver stains were prepared. No immunofluorescence slides were prepared as the hospital does not have facility for immunohistochemistry. Light microscopy revealed 25 glomeruli with diffuse endocapillary proliferation, fibro-cellular crescents in three glomeruli, wire loops in capillary tufts and mesangial matrix expansion (Figures 1 and 2) which are consistent with lupus nephritis class IV-S.

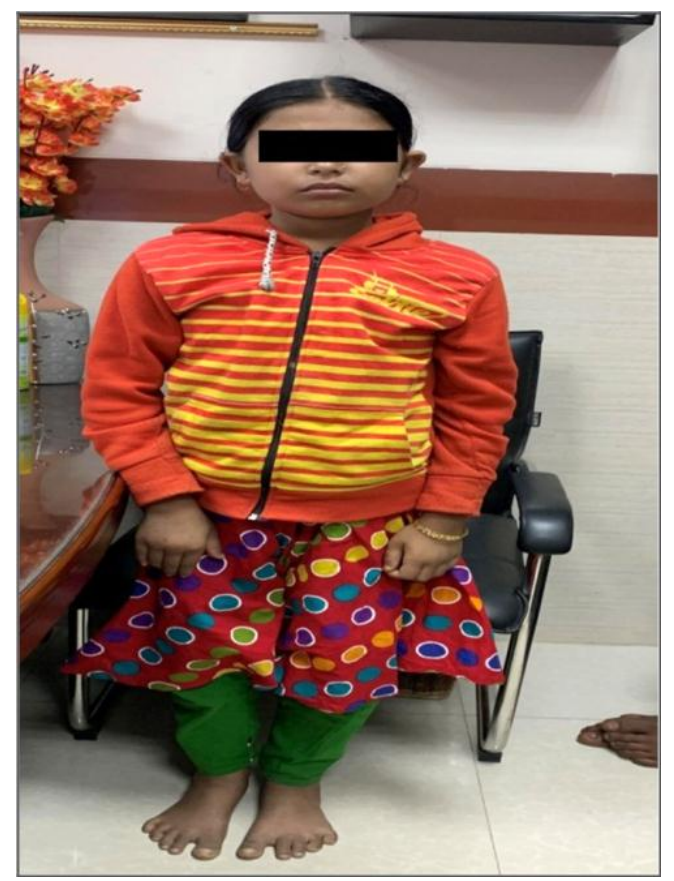




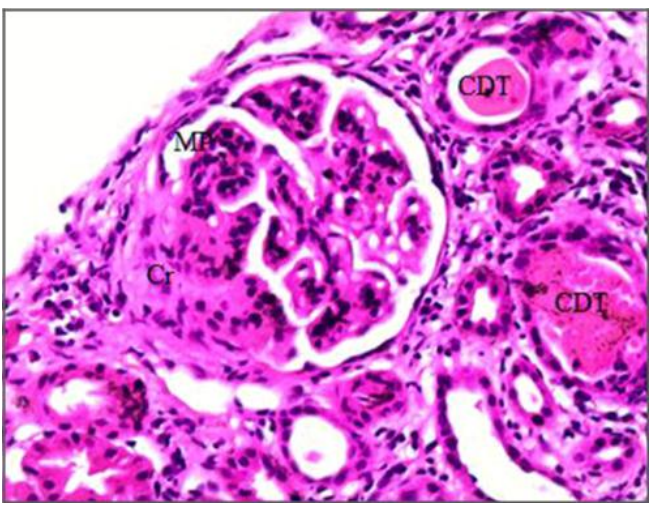

Figure 1: Glomerular lesion with fibro-cellular crescent (Cr) and mesangial proliferation (MP), CDT indicates cast in the tubules (Haematoxylin and Eosin stain; magnification $x$ 400)

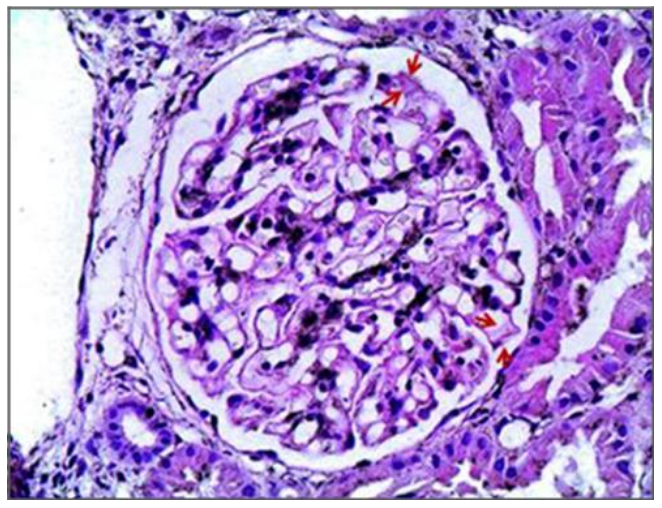

Figure 2: Double contouring (wire-loops) of glomerular basement membrane (arrows) (Periodic acid-Schiff (PAS) stain; magnification x 400)

She was given parenteral furosemide for oedema and hyperkalaemia and potassium was lowered to 5.4 after 5 days of diuretic treatment. The patient was given intrvenous methyl prednilone infusion for three consecuitive days followed by oral prednisolone $1 \mathrm{mg} / \mathrm{kg}$ body weight and oral Mycophenolate mofetil $500 \mathrm{mg}$ one tablet three times daily for lupus nephritis and her laboratory normalized after two months of treatment. She was also given atorvastatin for hypercholesterolemia. Her laboratory results after one month of treatment revealed neither protein nor red blood cells, serum albumin was $40 \mathrm{~g} / \mathrm{L}$, serum cholesterol was $5.9 \mathrm{mmol} / \mathrm{L}$ and serum creatinine was $0.9 \mathrm{mg} / \mathrm{dl}$.

\section{Discussion}

Systemic lupus erythematosus (SLE) is an autoimmune disease with variable manifestations. It is a heterogeneous disease of disordered immunity, with multi-system inflammatory involvement. Renal involvement is a frequent feature occurring in $40 \%-75 \%$ of patients, most often within five years of the disease onset. ${ }^{7,8,9,10}$

The course of lupus nephritis (LN) is highly variable and multiple clinical, serological, histopathological, and time-dependent factors are responsible for its ultimate prognosis. ${ }^{11,12}$

The clinical features of SLE have been extensively described from different geographical parts in the world, with some variations among different racial groups. The data on SLE among Bangladeshi patients are rare in the literature.

The present study which included both adult patients and children was carried out to observe the clinical profile and outcome of patients suffering from SLE in this southwestern region of Bangladesh. Mean age of the patients was $25.4 \pm 9.05$ years. Baqui et al showed the mean age of LN $26 \pm 11.97$ years. ${ }^{13}$

This is an autoimmune condition in which immune complexes play important role in causing tissue injury involving multiple organs and systems. ${ }^{14}$

Affected tissues in SLE show marked inflammation with deposition of antibodies and complements. The antibodies are autoantibodies directed against double stranded DNA of affected cells. Female are more affected with SLE and Africans arereported to be more commonly than other ethnic groups. ${ }^{15}$

Musculoskeletal and dermatological manifestations in the form of arthritis and discoid rashes are common presentation of SLE. ${ }^{16}$

These features were also reported by our patient. However the typical malar rashes of SLE were not reported. The patient also presented with pleural and pericardial effusion which are part of multi-organ 
involvement in patients with SLE. ${ }^{17}$

The diagnoses of SLE is usually made clinicallywith presence of at least four of the 11 American College of Rheumatology Classification criteria; malar rash, discoid rash, photosensitivity, oral ulcers, arthritis, serositis (pleural effusion,pericardial effusion), renal disorder, neurological disorder, haematological disorder, immunologic disorder and antinuclear antibodies. ${ }^{17}$ Our patient fulfilled the above criteria and the diagnosis of SLE with lupus nephritis was made. Management of lupus nephritis requires a biopsy to histologically classify the condition based on the extent, activity and chronicity. The extent of renal involvement is also assessed and provides a baseline subsequent follow. ${ }^{18}$

Lupus nephritis is histologically classified into six classes based on the International Society of Nephrology/ Renal Pathology Society classification. ${ }^{19}$ The six classes have been well described by Seshan \& Jennete $(2009)^{18}$, Our patient was classified as lupus nephritis class IV-S which signify segmental involvement of $>50 \%$ of the glomeruli examined histologically. Mycophenolate mofetil (MMF) with prednisolone has been associated with high remission rates when used as induction treatment for lupus nephritis. ${ }^{20}$ Pecoraro et al published an abstract of 14 children with lupus nephritis (mean age 12.4 years) with more than $3 \mathrm{gm}$ of proteinuria daily and normal renal function who were treated with IV methyprednisolone followed by MMF (mean dose $29 \mathrm{mg} / \mathrm{kg}$ daily) and oral prednisolone for 2 years.

This report describes the case of histologically proven lupus nephritis in a patient presenting with nephrotic syndrome at Community Based Medical College Hospital Bangladesh (CMCHB). It is therefore important to evaluate patients presenting with nephrotic syndrome for SLE serologically and then histologically for those with SLE to determine the extent of disease and appropriate treatment.

\section{References}

1. Appel GB, Radhakrishnan J, D'Agati V. In: The Kidney. 8. Brenner BM, editor. Philadelphia, PA: Saunders; 2007. Secondary glomerular disease; pp. 1067-1148.

2. Bertsias G, Gordon C, Boumpas DT. Clinical trials in SLE: lessons learned from the past as we proceed to the future-the EULAR recommendations for the management of SLE and the use of end-points in clinical trials. Lupus. 2008;17:437-442.

3. Mak A, Mok CC, Chu WP, To CH, Wong SN, Au TC. Renal damage in systemic lupus erythematosus: a comparative analysis of different age groups. Lupus. 2007;16:28-34.

4. Seligman VA, Lum RF, Olson JL, Li H,

Criswell $L A$. Demographic differences in the outcome of SLE nephritis: a retrospective analysis. Am J Med. 2002;112:726-729.

5. Cameron JS. Lupus nephritis. J Am Soc Nephrol. 1999;10:413-424.

6. Sigdel MR, Shah DS \&Raut KB. Lupus nephritis: a comprehensive review. Journal of Institute of Medicine. 2013;35, 58-70.

7. Walker WG, Solez K, Renal involvement in disorders of connective tissue. In: Early LE, Gottschalk CW, editors. Diseases of Kidneys. Boston: Little \& Brown. 1979; p. 1259-88.

8. Ballou SP, Khan MA, Kushner I. Clinical features of systemic lupus erythematosus: Differences related to race and age of onset. Arthritis Rheum. 1982;25:55-60.

9. Citera G, Wilson WA, Ethnic and geographic perspectives in SLE. Lupus. 1993;2:351-3.

10. Ward MM, Studenski S. Clinical manifestations of systemic lupus erythematosus. Identification of racial and socioeconomic influences. Arch Intern Med. 1991;91:343-53.

11. Mok CC. Prognostic factors in lupus nephritis. Lupus. 2005;14:39-44.

12. Baqui MN, Akhter S, Kabir E, Islam MS. A clinicopathological study on lupus nephritis; experience of 34 cases from Bangladesh. J Nephropharmacol. 2016;5:19-23.

13. Satish S, Deka $P$, Shetty MS. A clinicopathological study of lupus nephritis based on the International Society of Nephrology-Renal Pathology Society 2003 classification system. J Lab Physicians. 2017;9:149-55. 
14. Tsokos GC. Systemic lupus erythematosusmechanism of disease. New England Journal of Medicine. 2011;365, 2110-2121.

15. Carey $R$, Simmons $S$, Malherbe $M$, Jansen van Rensburg BJ \&Joubert G. Clinical features of patients with systemic lupus erythermatosus (SLE) attending SLE outpatient clinic at Universitas Hospital in Bloemfontein, South Africa. South Africa Family Practice. 2008;50,68.

16. Dhakal SS, Sharma KS, Bhatta N, Bhattarai S, Karki S, Shrestha S, Rijal S\&Karkil P. Clinical features and histological patterns of lupus nephritis in Eastern Nepal. Saudi Journal of Kidney Diseases and Transplantation. 2011;22, 377-380.

17. Gill JM, Quisel AM, Rocca PV \& Walters DT.

Diagnosis of systemic lupus erythematosus. American Family Physician. 2003;68, 2180 2186.

18. Seshan SV \&Jennete C. Renal disease in systemic lupus erythematosus with emphasis on classification of lupus glomerulonephritis. Archives of Pathology and Laboratory Medicine. 2009;133, 233-248

19. Markowitz GS \&D'Agati VD. The ISN/RPS 2003 classification of lupus nephritis: Anassessment at 3 years. Kidney International. 2007;71, 491-495.

20. Pecoraro $C$, Malgieri G, D'Armiento $M$, et al. Treatment of childhood lupus nephritis with mycophenolatemofetil: clinical and histopathological study. J Am Soc Nephrol. 2005;16:557A. 